\title{
IDENTIFICACIÓN DE UNA NUEVA POBLACIÓN DE ALTO RIESGO NEONATAL CON CURVAS DE CRECIMIENTO INTRAUTERINO PROPIAS
}

\author{
Manuel Ticona Rendón'; Diana Huanco Apaza ${ }^{2}$
}

\section{RESUMEN}

Introducción: El peso de nacimiento en relación con la edad gestacional tiene valor pronóstico y sirve para el manejo clínico del recién nacido. La OMS recomienda patrones de crecimiento fetal en los centros perinatológicos, pues se detectan diferencias según países y condiciones de vida.

Material y Métodos: Se realizó enrolamiento prospectivo de RN vivos sucesivos, técnica de pesaje, diagnóstico de edad gestacional por FUM; exclusión de patología materna y fetal; descarte de desnutrición materna; se utilizó el Sistema Informático Perinatal (CLAP-OPS/OMS) y otros sistemas computacionales; test estadísticos necesarios.

Resultados: 8,735 RN vivos fueron seleccionados en 6 años (1993-1998), su peso promedio de nacimiento fue 3297-3619 gr. y el percentil 10 de peso de nacimiento fue 2710-3085 gr. a las 38 y 41 semanas respectivamente. Estos datos son significativamente diferentes a los de Lubchenco. La multiparidad y talla materna alta y el sexo fetal masculino produjeron peso de nacimiento significativamente mayor.

La Curva de Crecimiento Fetal obtenida diagnostica 9.2\% RN-PEG en riesgo us, $1.2 \%$ que diagnostica la Curva Lubchenco $(p<0.0000001)$. El $8 \%$ de los Nuevos RN-PEG identificados demostró mayor riesgo de mortalidad (8/1321 us 24/11679; $p<0,01$ ), de morbilidad 218/1321 us 339/11679, $p<0.01)$ que los $R N-A E G$.

Conclusiones: Se obtuvieron Curva de Crecimiento Fetal en población tacneña bien seleccionada y factores de corrección por paridad y talla materna y sexo fetal. Se demostró su diferencia con Curvas de Lubchenco, identificando una nueva población de riesgo neonatal (RNPEG) con mayor morbi-mortalidad. Se recomienda su uso en los establecimientos de Salud del país.

1 Profesor Principal de la FACM. Ex Jefe de Neonatologia del Hospital Hipólito Unanue Tacna

2 Jefe de Epidemiologia del Hospital Hipólito Unanue de Tacna. 


\section{ABSTRACT}

Introduction: The weight from birth in relation to the age gestacional he/she has value presage and it is good for the clinical handling of the recently born one. The OMS recommends patterns of fetal growth in the centers perinatológicos, because differences are detected according to countries
and conditions of life.

Material and Methods: He/she was carried out prospective enrolamiento of successive alive $R N$, pesaje technique, diagnosis of age gestacional for FUM; exclusion maternal and fetal pathology; discard of maternal malnutrition; the Computer System Perinatal was used (CLAP-OPS/OMS) and other systems computacionales; necessary statistical test.

Results: 8,735 alive $R N$ was selected in 6 years (1993-1998), its weight averages from birth it was 3297-3619 gr. and the percentile 10 of weight from birth were 2710-3085 gr. to the 38 and 41 weeks respectively. These data are significantly different to those of Lubchenco. The multiparidad and high maternal size and the masculine fetal sex produced weight from birth significantly bigger.

The obtained Curve of Fetal Growth diagnoses 9.2\% RN-PEG in risk vs, $1.2 \%$ that diagnoses the Curve Lubchenco ( $p<0.0000001) .8 \%$ of the New identified RN-PEG demonstrated bigger risk of mortality (8/1321 vs 24/11679; $p<0,01)$, of morbilidad $218 / 1321$ us 339/11679, $p<0.01$ ) that the
RN-AEG.

Conclusions: Curve of Fetal Growth was obtained in population well selected tacneña and correction factors by parity and maternal size and fetal sex. Their difference was demonstrated with Curved of Lubchenco, identifying a new population of risk neonatal (RN-PEG) with more morbi-
mortality. Their use is recommended in the establishments of Health of the country.

\section{INTRODUCCIÓN}

La relación entre el peso de nacimiento y la edad gestacional (EG) tiene mayor valor pronóstico que el peso de nacimiento por sí solo.

La clasificación del RN en uno de los 9 grupos de Lubchenco, según su peso esté bajo el percentil 10, sobre el 90 ó entre ambos, es recomendada y usada ampliamente con fines pronósticos y de manejo clínico. Requiere utilizar una tabla patrón de crecimiento intrauterino (CIU) apropiada, que la OMS recomienda confeccionar en los Centros Perinatológicos.

En el Hospital Hipólito Unanue de Tacna (HHUT) se utilizan las Curvas de Crecimiento Intrauterino (CCIU) de la Dra. Lubchenco (25), como lo hacen en la mayoría de Hospitales del Perú.

En la práctica clinica diaria hemos observado que los RN GEG determinados con las Curvas de Lubchenco, en gran mayoría no presentan ninguna patologia; por el contrario, hay un grupo de RN adecuados para la edad gestacional (AEG) que se comportan clínicamente como RN PEG, por lo que se intuye que probablemente se ha sobredimensionado ha los RN GEG y se ha subdimensionado a los RN PEG, demostrando que las curvas de Lubchenco son un patrón poco exigente para nuestros RN.

La importancia de contar con tablas propias de Crecimiento Intrauterino que permitan establecer el peso en relación con la edad gestacional, es sin duda el reto más importante con el que se encuentran profesionales inmersos en la atención y cuidados del binomio Madre-Niño.

Las Curvas de Crecimiento Intrauterino (CCIU) de Lubchenco (EEUU) (10) tiene limitaciones en su uso derivadas, en parte, de haber sido confeccionadas en Denver Colorado en EE.UU., en una población ubicada en altura y con determinadas caracteristicas raciales.

Por estas razones es necesario confeccionar CClU que sean las más apropiadas a la población en que se apliquen, usando una metodología concordante con recomendaciones nacionales e internacionales, así como su aplicación para la identificación real de los grupos de alto riesgo: PEG y GEG, los que están asociados a mayor morbi-mortalidad. 
En un sentido práctico, con este estudio se pretende identificar un grupo de $\mathrm{RN}$ de riesgo que antes no era considerado como tal, y encontrar sus factores perinatales asociados, lo que nos permitirá realizar acciones concretas para disminuir las tasas de morbi-mortalidad neonatal en nuestro medio.

\section{MATERIAL Y MÉTODOS}

Es un estudio analítico, transversal, utilizando datos pre recolectados, que tiene la finalidad de desarrollar y adaptar una metodología estandarizada de clasificación del estado nutricional del recién nacido.

PARA LA CONFECCIÓN DE LAS CCIU: Se incluyeron a todos los RN de 26 a 43 semanas de gestación durante el período indicado. Las madres y sus RN debieron presentar ciertos requisitos:

- RN de embarazos únicos, sin malformaciones congénitas, cuyas madres no presentan patología durante el embarazo, sin sufrimiento fetal crónico, ni retardo de crecimiento intrauterino ni infecciones intrauterinas

- Madres sin hábitos nocivos: fumadoras, alcohólicas.

- Sin desnutrición materna (Indice de Quetelet > 18)

- Madres con fecha de última menstruación (FUM) confiable.

En este período de observación nacieron 15,739 RN vivos, y $8,841(56.17 \%)$ de ellos cumplieron el criterio de selección, dando origen a los datos presentados.

PARA LA IDENTIFICACIÓN DE LA NUEVA POBLACIÓN DE RIESGO, se ha considerado el universo de $15,739 \mathrm{RN}$ vivos. A todos los $\mathrm{RN}$ vivos se llevaron a las CCIU propias que hemos confeccionado, identificándose como población de riesgo a los $\mathrm{RN}$ que se encuentren por debajo del percentil 10 denominándolos NUEVOS PEG, los cuales se subdividieron en tres grupos: PEG leve, moderado y severo, según esté bajo el percentil 10, el 5 ó el 2.5 respectivamente.

La recolección de la información se realizó en el banco de datos del Sistema Informático Perinatal (CLAP OPS/OMS), que tiene buena calidad de información.
Se procedió a la construcción de las Curvas de Crecimiento Intrauterino Propias con el Programa Excel, utilizando el ajuste Polinomial de Tercer Orden.

Para el análisis estadístico de los datos se utilizaron tablas con percentiles $(2.5,5,10,50,90)$, promedios y desviaciones estandar, para peso, por cada semana de gestación.

Se elaboraron curvas de crecimiento intrauterino de peso, en relación con la EG, con los percentiles obtenidos.

Se elaboraron curvas de factores de correción según sexo fetal, paridad y talla materna.

Se compararon los promedios de peso por edad gestacional de las Curvas de Lubchenco con las

- nuestras, y se determinó a través de pruebas estadísticas a partir de qué semanas de gestación habia diferencias estadísticamente significativas, identificando de esta manera la NUEVA POBLACIÓN PEG DE ALTO RIESGO NEONATAL.

En la comparación estadística con las CCIU de Lubchenco se considera $<$ significativo $p<0,05$.

Se sobrepondrán las CCIU de Lubchenco y las nuestras, para la cuantificación de los RN PEG y GEG.

\section{DISCUSIÓN Y COMENTARIOS}

\section{CURVAS DE CRECIMIENTO INTRAUTERINO DE ACUERDO CON EL PESO DE NACIMIENTO:}

Se seleccionaron 8,735 recién nacidos $(R N)$ vivos de ambos sexos, que cumplieron las condiciones de selección descritas. Se obtuvieron percentiles y promedios de peso, por semana de edad gestacional.

El Gráfico N ${ }^{0} 1$ presenta los percentiles ajustados $2.5,5,10,50$ y 90 del Peso al Nacer entre las semanas 26 y 43 de EG. En cada caso se ajustó un polinomio de tercer orden.

Además de considerar los percentiles 10,50 y 90 , se calcularon los percentiles 2.5 y 5 para clasificar al RN PEG en: leve, moderado o severo, según esté bajo el percentil 10 , el 5 o el 2.5 respectivamente; con la finalidad de protegerlo con medidas progresivamente más importantes según su gravedad. 


\section{FACTORES DE CORRECCION:}

Se realizó el análisis de factores que influyen en el CIU: sexo fetal, paridad materna y talla materna.

Se estudiaron por separado los RN masculinos y femeninos y los hijos de primigestas y de multíparas, se expresaron los datos y se usaron pruebas estadísticas apropiadas según se especifica en resultados.

Hubo 3240 primigestas y 4150 multíparas, 4568 RN masculinos y $4167 \mathrm{RN}$ femeninos. Se obtuvieron promedios de peso por cada EG por paridad materna y sexo fetal.

Las diferencias de peso entre RN femeninos y masculinos y de primigestas y multíparas son pequeñas, pero estadisticamente significativas entre las semanas 38 a 42 y 37 a 42 respectivamente, con una $p<0.01$ (prueba T de Student).

Los RN masculinos tuvieron un PN promedio superiores en 113 a $150 \mathrm{~g}$. a los RN femeninos y los hijos de multíparas presentaron PN promedio 192 a $238 \mathrm{~g}$. mayores que los de primigestas en las EG mencionadas, en que hubo significación estadística.

Las diferencias de peso según sexo del RN y paridad materna, por cada semana de gestación en las que existe significancia estadística, respecto a la mediana, se muestran en la tablas $N^{0} 1$ y 2 y gráficos $\mathrm{N}^{\circ} 2$ y 3 respectivamente.

Además de la paridad materna y el sexo fetal, influyó de idéntica manera la talla materna en 8735 madres que dieron a luz $\mathrm{RN}$ vivos que se cumplieron las condiciones de selección, la talla materna fue consignada en 5833 casos $(66,8 \%)$. El promedio de la talla materna fue $155+6.1 \mathrm{~cm}(x+D E)$. De acuerdo con la desviación estándar, el grupo de madres se subdividió en 3 grupos, madres con talla igual o mayor de $162 \mathrm{~cm}$, de 149 a $161 \mathrm{~cm}$ y con talla igual o menor a $148 \mathrm{~cm}$. Los 3 grupos tuvieron RN con peso y talla significativamente relacionados en forma directa a la talla materna, se utilizó el test de hipótesis para promedios, $(p<0.01)$. El perímetro cefálico y la edad gestacional no fueron influidos por la talla materna (Tabla $\mathrm{N}^{\circ} 3$ ).

En sintesis: las madres con talla menor del promedio $(155 \mathrm{~cm})$ tienen $\mathrm{RN}$ con $\mathrm{PN}$ inferior que las madres con talla mayor que el promedio $(p<0.01$, prueba T de Student). Los resultados de este estudio prospectivo demuestran una correlación importante entre la talla materna y los promedios de peso del
RN. Ello permite proponer la aplicación de un factor de corrección al PN, antes de clasificar al niño como PEG. De los resultados expuestos se deduce que la talla materna influye sobre el peso del nacimiento y que al aplicar el factor de corrección se clasifica más apropiadamente al RN, con el objeto de evaluar el embarazo de alto riesgo y proteger al niño PEG, el cual tiene mayor riesgo perinatal.

Basados en los resultados aqui encontrados, se recomienda clasificar al RN según EG, incluyendo los factores de corrección según paridad, talla materna y según sexo fetal. Otros autores han encontrado similares datos entre los cuales podemos citar a la Dra. Gabriela Juez $(16,17)$, de tal modo que los factores señalados deben ser tomados en cuenta en estudios de investigación.

Para mayor precisión, en el trabajo clínico se diseñaron los recuadros del Gráfico $N^{0} 1$, que permite hacer las correcciones necesarias según sexo fetal, talla y paridad materna, usando un solo gráfico patrón. En estos recuadros se señalan las correcciones respecto a la mediana que es necesario practicar al clasificar al RN según su sexo, paridad y talla de su madre en las EG en que estos factores marcan una diferencia significativa ( 38 a 42 semanas), en el caso de la talla materna la corrección se calculo en una recta de regresión.

\section{COMPARACIÓN DE CURVAS DE TACNA CON LAS DE LUBCHEÑCO}

En el Perú se usan de forma prevalente las Curvas de Lubchenco; sin embargo, el uso de esta curva tiene variadas limitaciones que han sido señaladas por diversos autores. Por otro lado, hay también problemas derivados del desconocimiento que en esa época existía de algunos factores de RCIU que impidieron adecuada selección de los RN. Tampoco se conocía en detalle los métodos para evaluar la EG de acuerdo a signos físicos y neurológicos ni existían las facilidades actuales para el procesamiento computacional de datos. Por esta razón, se han hecho y se continúan haciendo curvas de crecimiento que sean las más apropiadas para la población en que se apliquen.

La curva de peso confeccionada en el presente estudio con RN normales seleccionados en forma prospectiva, usando una metodologia concordante con recomendaciones internacionales, demuestra ser significativamente distinta a la de Lubchenco, ya que 
los promedios de peso fueron significativamente mayores que los comunicados por Lubchenco entre 37 a 42 semanas de EG (t de Student, Tabla $N^{\circ} 4$ ).

Comparando los percentiles de peso de nuestro estudio con los de Lubchenco, afirmamos que los límites del percentil 10 y 90 de Tacna no coinciden con los de Lubchenco; así, por ejemplo, en la semana 40 , que es la más representativa, encontramos que en las CCIU de Tacna es de 2983 y 4062 respectivamente y en las de Lubchenco 2560 y $3700 \mathrm{~g}$ respectivamente, lo que demuestra que las nuestras son mayores.

Estos hallazgos confirman la necesidad de contar con información nacional y local sobre esta materia, construyendo curvas para valorar $\mathrm{CIU}$ según peso.

\section{IDENTIFICACIÓN DE UNA NUEVA POBLACION DE RIESGO}

La identificación oportuna de los factores de riesgo que inciden en la morbi-mortalidad del $\mathrm{RN}$ es un objetivo primario de la Perinatologia moderna. Permite prevenir problemas o preparar con anticipación el tratamiento oportuno de ellos. EI RCIU es reconocido como un factor importante de riesgo perinatal.

Para evaluar el ClU se usan curvas y tablas que establecen los rangos normales para el peso del RN por cada semana de EG, ya que el peso es el indice más sensible como factor determinante de riesgo, y el que con mayor frecuencia se altera.

Se estudio y comparó la distribución de todos los RN en AEG, PEG y GEG, aplicando sucesivamente las tablas de peso Intrauterino Lubchenco y la nuestra. Se identificó el grupo de RN-PEG adicionales, restando el número de RN-PEG obtenidos con la curva de Lubchenco al de RN-PEG obtenidos con nuestra curva. La identificación de este grupo se encuentra entre los percentiles 10 de la curva de Lubchenco y la nuestra, al hacer una sobreposición gráfica de ambas curvas (Gráfico $N^{\circ} 5$ ). Este grupo será denominado en adelante "NUEVOS RN PEG».

Hemos cuantificado el aumento en el porcentaje de RN PEG, el que se adicionan al de Lubchenco al aplicar la nueva curva, y que eran considerados RNAEG cuando se usaba la curva de Lubchenco. Con esto hemos identificado un nuevo grupo de RN de mayor riesgo que antes no era considerado como tal.
La variación en la distribución de los RN clasificados por peso y EG al aplicar la curva de Lubchenco fue la siguiente: los RN GEG disminuyeron de un $15,6 \%$ a un $9,5 \%$; Ios RN AEG mantuvieron un porcentaje semejante de $83,2 \%$ y $81,3 \%$, y los RN PEG aumentaron de un $1,2 \%$ a un $9,2 \%$ (Gráfico N ${ }^{\circ} 5$ ). El cambio en el porcentaje de RN GEG y de RN PEG fue estadisticamente significativo (con una $p<0.000000001$ ). El número de RN-PEG usando nuestra curva fue de 1448 y de 189 usando la de Lubchenco. La diferencia entre estos dos grupos es de 1259 RN ( $8 \%$ de la población total) que corresponde al grupo que hemos denominado RN Nuevos PEG (Tabla N ${ }^{0} 6$ ). De un estudio de 11543 RN en el Hospital de la Universidad Católica de Chile, utilizando las curvas de Lubchenco se encontró $2 \%$ de RN-PEG, pero utilizando las CCIU propias de Chile, cuyos autores están liderados por la Dra. Gabriela Juez, este porcentaje se incrementó hasta llegar a un $11 \%$ (14).

Con estas curvas hemos obtenido un aumento de RN-PEG de un $1,2 \%$ a un $9,2 \%$ en niños de más de 36 semanas de edad gestacional. Queda para una investigación posterior el de determinar qué pasan con los niños menores de 36 semanas de EG. Uno de los problemas para esto es la dificultad de obtener un número suficiente de prematuros «normales» para completar nuestras curvas de CIU. Además, está el hecho de que el número de RNPEG es escaso en estas edades gestacionales, lo que dificulta las comparaciones estadísticas.

Creemos que los resultados más relevantes de esta investigación son los referentes a la morbimortalidad y antecedentes perinatales de los RN que hemos llamado Nuevos PEG. En nuestro hospital usando las curvas de Lubchenco, si bien es cierto que obteniamos un porcentaje bajo (1.2\%) de RN-PEG, sabiamos por nuestras estadísticas de mortalidad que se trataba de un grupo de muy alto riesgo con una letalidad de $7.41 \%$ (37), 20 veces más alta que la de los RN-AEG.

Con nuestras curvas obtenemos un aumento de un $8 \%$ de los RN PEG, pero quedaba la pregunta si este grupo adicional de PEG era también un grupo de alto riesgo de morbi-mortalidad, que es uno de los elementos más importantes en darle relevancia clínica a una curva de peso intrauterino. Los estudios nacionales efectuados al respecto sólo señalan que los percentiles de sus curvas de peso intrauterino, en especial el percentil 10, eran más altos que los de Lubchenco, pero no habían cuantificado en cuánto 
aumentaban los RN-PEG, ni determinado si este aumento se hacia con RN también de alto riesgo. En nuestro estudio queda demostrado que el grupo de RN PEG tienen efectivamente un riesgo mayor de morbi-mortalidad, que los RN-AEG. De tal manera que su clasificación como PEG no es sólo una mera distribución estadística sino que corresponde a un comportamiento clínico distinto.

La mortalidad infantil en el Perú ha disminuido ostensiblemente en los últimos años, pues hace 10 años ocupábamos los últimos lugares sólo antes de Haití y Bolivia, gracias principalmente a los programas Preventivo-Promocionales, tales como los Programas del PAI, EDA, IRA principalmente; actualmente nuestra Tasa de Mortalidad de $46 \times 1000$ nv nos permite ocupar el promedio entre los países latinoamericanos; es decir, se ha disminuido la mortalidad infantil a expensas de mortalidad postneonatal. La mortalidad neonatal ocupa asi un lugar preponderante dentro de la mortalidad infantil en la actualidad llegando a un $50 \%$ de ésta, por lo que es importante, para mejorar estas cifras, investigar en forma más fina los $\mathrm{RN}$ de riesgo alto identificando no sólo aquellos que presentan muy alto riesgo, como es el caso del $1,2 \%$ de RN-PEG que se detecta con las curvas de Lubchenco, sino los de un moderado riesgo que serían los PEG adicionales (Nuevos PEG), resultantes de la aplicación de nuestras curvas a la realidad local. Estos resultados son semejantes al estudio realizado por la Dra. Juez, donde también ha encontrado una mayor morbi-mortalidad de este Nuevo Grupo de RN-PEG (19).

Esto debería llevar a tomar acciones, tanto preventivas como de manejo clínico concordante con su riesgo, por eso la importancia de subdividir los RN PEG en leves, moderados y severos de acuerdo con que estén bajo el percentil 10,5 y 2,5 respectivamente. Sería importante avanzar en esta línea en investigaciones futuras que determinen los riesgos y causas de morbi-mortalidad en estos subgrupos de RN-PEG.

Nuestro estudio está hecho en una población de nivel socioeconómico medio y bajo, que es probablemente el término medio de la población peruana. Por esta razón nos parece válido pensar que si se aplicaran nuestras CCIU a la totalidad de los RN peruanos, el aumento de RN PEG seria igual o mayor al encontrado en este trabajo. En 1996 nacieron en el Perú 615000 RN (13). Un cáiculo aproximado nos da alrededor de $565800 \mathrm{RN}$ de más de 36 semanas. Aplicando las cifras de este trabajo tendríamos un $8 \%$ de RN-PEG que, usando las curvas de Lubchenco, no fueron diagnosticados como tales: esto corresponde a 45264 niños.

Aplicando la cifra de mortalidad $(0,61 \%)$ que hemos encontrado para este grupo tendríamos 276 niños fallecidos. Si con medidas preventivas y de buen manejo neonatal se llegara a una cifra de mortalidad semejante a los RN-AEG $(0,21 \%)$, la cifra de niños fallecidos bajaría a 95. Algo semejante podría lograrse con la morbilidad al nacer. Estas consideraciones son solamente una hipótesis que necesita comprobación. Lo más probable es que nunca se llegue a bajar la morbi-mortalidad a las cifras recién mencionadas. Sin embargo, hemos querido hacerlo para poner en relieve la importancia que podrían tener en salud pública los resultados aquí presentados.

\section{RESULTADOS}

Tabla 1. Factores de corrección según peso del RN.

\begin{tabular}{|c|c|c|c|c|c|}
\hline EG & X Peso & Masculino & $(+)$ & Femenino & $(-)$ \\
\hline 38 & 3297 & 3366 & +69 & 3216 & -81 \\
39 & 3410 & 3477 & +67 & 3342 & -68 \\
40 & 3514 & 3570 & +56 & 3457 & -57 \\
41 & 3619 & 3679 & +60 & 3552 & -67 \\
42 & 3681 & 3745 & +64 & 3607 & -74 \\
\hline
\end{tabular}

Tabla 2. Factores de correción según paridad materna.

\begin{tabular}{|c|c|c|c|c|l|}
\hline EG & X Peso & Multipara & $(+)$ & Primigesta & \multicolumn{1}{|c|}{$(-)$} \\
\hline 38 & 3297 & 3389 & +92 & 3198 & -99 \\
39 & 3410 & 3506 & +96 & 3305 & -105 \\
40 & 3514 & 3607 & +93 & 3415 & -99 \\
41 & 3619 & 3725 & +106 & 3507 & -112 \\
42 & 3681 & 3757 & +76 & 3519 & -162 \\
\hline
\end{tabular}

Tabla 3. Crecimiento fetal y talla materna.

\begin{tabular}{|c|c|c|c|c|c|}
\hline \multicolumn{5}{|c|}{5.833 Madres } & Talla $155+6.1$ \\
\hline $\begin{array}{c}\text { Talla } \\
\text { Materna }\end{array}$ & $\mathrm{N}$ & $\begin{array}{c}\text { Peso Nac } \\
\mathrm{X}+\mathrm{DE}\end{array}$ & $\begin{array}{c}\text { Talla NacX } \\
\text { DE }\end{array}$ & P.Cefálico & EG X \\
\hline$>162 \mathrm{~cm}$ & 720 & $3516+464$ & $50.98+2.27$ & $349+16.0$ & 39.5 \\
\hline $149-161 \mathrm{~cm}$ & 4411 & $3434+464$ & $50.66+2.21$ & $346+16.4$ & 39.4 \\
\hline$<148 \mathrm{~cm}$ & 702 & $3407+428$ & $50.51+1.92$ & $347+15.5$ & 39.6 \\
\hline
\end{tabular}


Tabla 4. Promedios de peso al nacer. Tacna-Lubchenco.

\begin{tabular}{|c|c|c|c|c|c|c|c|}
\hline \multirow{2}{*}{ EG } & \multicolumn{3}{|c|}{ TACNA } & \multicolumn{3}{c|}{ LUBCHENCO } & \multirow{2}{*}{$\mathrm{P}$} \\
\cline { 2 - 7 } SEM & $\mathrm{N}$ & $\mathrm{X}$ & $\mathrm{DE}$ & $\mathrm{N}$ & $\mathrm{X}$ & $\mathrm{DE}$ & \\
\hline 36 & 67 & 2747 & 421 & 202 & 2710 & 519 & $\mathrm{NS}$ \\
37 & 283 & 3126 & 399 & 372 & 2900 & 451 & SIGN. \\
38 & 1190 & 3297 & 387 & 636 & 3030 & 451 & SIGN. \\
39 & 2403 & 3410 & 387 & 1010 & 3140 & 402 & SIGN. \\
40 & 3208 & 3514 & 403 & 1164 & 3220 & 396 & SIGN. \\
41 & 1268 & 3619 & 405 & 632 & 3290 & 396 & SIGN. \\
42 & 197 & 3681 & 438 & 336 & 3300 & 423 & SIGN. \\
\hline
\end{tabular}

Tabla 5. Identificación del nuevo grupo PEG.

\begin{tabular}{|c|c|c|c|c|}
\hline \multirow{2}{*}{$\begin{array}{c}\text { RELACION } \\
\text { PESO / EG }\end{array}$} & \multicolumn{2}{|c|}{ LUBCHENCO } & \multicolumn{2}{c|}{ TACNA } \\
\cline { 2 - 5 } & $N^{0}$ & $\%$ & $N^{0}$ & $\%$ \\
\hline PEG & 165 & 1.2 & 1,321 & 9.2 \\
\hline AEG & 10,926 & 83.2 & 11,679 & 81.3 \\
\hline GEG & 2,047 & 15.6 & 1,366 & 9.5 \\
\hline TOTAL & 13,138 & 100.0 & 13,138 & 100.0 \\
\hline
\end{tabular}

\section{CONCLUSIONES}

1. Durante el período de enero de 1993 a diciembre de 1998 nacieron en el Hospital de Apoyo «Hipólito Unanue» de Tacna 15739 RN vivos, de los cuales 8735 (55.5\%) fueron RN sanos, sin ningún factor relacionado con Retardo de Crecimiento Intrauterino, con el que se confeccionaron las Curvas de Crecimiento Intrauterino para $\mathrm{RN}$ tacneños.

2. Además de considerar los percentiles 10,50 y 90 , se calcularon los percentiles 2,5 y 5 para clasificar al RN PEG en: leve, moderado o severo, según esté bajo el percentil 10, el 5 o el 2,5 respectivamente, según su gravedad.

3. Las diferencias de peso entre $R N$ femeninos y masculinos y de primigestas y multíparas fueron pequeñas, pero estadísticamente significativas entre las semanas 38 a 42 para sexo y 37 a 42 para paridad.

4. Los RN masculinos tuvieron un PN promedio superiores en 113 a $150 \mathrm{~g}$ a los RN femeninos y los hijos de multíparas presentaron PN promedio 192 a $238 \mathrm{~g}$ mayores que los de primigestas en las EG mencionadas, en que hubo significación estadistica.

5. El promedio de la talla materna fue $155+6,1 \mathrm{~cm}$ $(X+D E)$. El peso y talla de los $R N$ tuvieron relación directa con la talla materna. El perímetro cefálico y la edad gestacional no fueran influidos por la talla materna.

6. Se recomienda clasificar al $R N$ según $E G$, incluyendo los factores de corrección según paridad, talla materna y sexo fetal en las EG en que estos factores marcan diferencia significativa ( 38 a $42 \mathrm{sem}$ ).

7. Los percentiles 50 para talla, perímetro cefálico $e$ Indice Ponderal para EG de 40 semanas fueron 51,97 ; 341 y 2,58 respectivamente.

8. La curva de peso de RN tacneños es distinta a la de Lubchenco, ya que los promedios de peso fueron significativamente mayores que los comunicados por Lubchenco entre 37 a 42 semanas de EG.

9. Los percentiles 10 y 90 de las CCIU de Tacna no coinciden con los de Lubchenco, así en la semana 40 para la CCIU de Tacna los límites son de 2983 a $4062 \mathrm{~g}$ y en los de Lubchenco de 2560 a $3700 \mathrm{~g}$, 10 que demuestra que los de Tacna son mayores.

10. Las Curvas de Talla de Tacna y Lubchenco no coinciden, los percentiles 10 y 50 de Tacna coinciden con los de 50 y 90 de Lubchenco respectivamente. En la semana 40 los percentiles 10,50 y 90 de Tacna son de 48,$23 ; 51,97$ y $54,63 \mathrm{~cm}$ y los de Lubchenco 45,$4 ; 48,9$ y $52,1 \mathrm{~cm}$.

11. En las curvas de Perímetro Cefálico, los percentiles 10 y 50 de Tacna coinciden con los de Lubchenco pero nuestro percentil 90 está muy por encima del 90 de Lubchenco. Así, en la semana 40 el PCN de las curvas de Tacna fue 312,$4 ; 341,0$ y $389,1 \mathrm{~mm}$ y el de Lubchenco 315,$0 ; 335,0$ y 356,5 $\mathrm{mm}$ respectivamente.

12. La variación en la distribución de los RN clasificados por peso y EG al aplicar la curva de Lubchenco y la de Tacna fue la siguiente: los RN GEG disminuyeron de un $15,6 \%$ a un $9,5 \%$; los RN AEG mantuvieron un porcentaje semejante de $83,2 \%$ y $81,3 \%$ y los RN PEG aumentaron de un $1,2 \%$ a un $9,2 \%$. El cambio en el porcentaje de RN GEG y de RN PEG fue estadisticamente significativo.

13. El número de RN-PEG, usando nuestra curva, fue de 1448 y de 189 usando la de Lubchenco. La diferencia entre estos dos grupos es de 1259 RN ( $8 \%$ de la población total) que corresponde al grupo denominado Nuevos RN-PEG. 


\section{RECOMENDACIONES}

1. Al comprobarse científicamente que las CCIU de Lubchenco no son adecuadas para nuestro medio, es imperativo la utilización de las CCIU de Tacna en todos los establecimientos de salud de nuestra Región, como un instrumento necesario para determinar los grupos de riesgo neonatal y asi

\section{REFERENCIAS BIBLIOGRÁFICAS}

Bailar, J.; Mosteller, F.: «La información Estadistica que Deben Proporcionar los Artículos Publicados en Revistas Médicas». Bol.Of.Sanit.Panam. 108(4), 1990.

Balcázar, H.; Haas, J.: «Tipos de Retardo del Crecimiento Intrauterino y Mortalidad Neonatal Precoz en Recién Nacidos de México». Bol.Of.Sanit.Panam. 11(5), 1991.

Beca, J.; Rizardini, M.; Weldt, E.; «Talla, Peso y Perímetro Craneano Según Edad Gestacional, en Recién Nacidos de menos de 35 semanas». Rev.Chil.Pediatr. 60(1); 1-5, 1989.

Bianchi, R.; y Cols.: «Características Materno-Neonatales del Retardo de Crecimiento Intrauterino en la Gestación de Término Según Curva Nacional de Crecimiento Intrauterino». Rev.Chil.Obstet.Ginecol, 56(6): 420-427, 1991.

Brenner, W.; Edelman, D.: «A Standard of Fetal Growth for the United Satated of American. Am.J.Obstet.Gynecol. Vol.126, No5. November, 1976.

Costa, R.: "El Recién Nacido en el Hospital de Belen de Trujillo". En: Oliveros, M.: Medicina Fetal y del Recién Nacido. CONCYTEC. 1997.

Cusminsky, M.; Moreno, E.; Suarez, E.: «Crecimiento y Desarrollo». Public. Científica $N^{0} 510$. Organización Panamericana de Salud. 1988.

Espinoza, R.; Goméz, R.: «Manejo clínico y Resolución del Parto con Retardo de Crecimiento Intrauterino». Rev. Chil.Obstet.Ginecol. 56(1): 55-63, 1991.

Fescina, R. y col.: «Vigilancia de Crecimiento Fetal». Pub.Cientif. del CLAP N1 1261. Uruguay. 1992.

Gayán, P. Y Cols.: «Retardo de Crecimiento Intrauterino». Rev.Chil.Obstet. Ginecol. 55(3):143-151, 1990

Gonzales, X.; Faneite, P.; Salazar, G.: «Retardo de Crecimiento Intrauterino (RCIU): Incidencia y Repercusión Perinatal». Rev.Obst. Gin. Venezuela. 48:15-18, 1988.

Guayasamin, O. y Cols.: «Crecimiento Fetal Humano Valorado por Indice Antropometricos». Pub.Cientif. del CLAP N1 1016. Uruguay. 1984.

Instituto Nacional de Estadística e Informática: «Encuesta Demográfica y de Salud Familiarm. Lima, Junio 1997.

Juez, G. y cols: «Crecimiento Intrauterino en Recién Nacidos Chilenos de Clase Media». Rev. Chil.Pediatr. 60(4); 198-202, 1989.

Juez y cols.: «Talla, Circunferencia Craneana e Indice Ponderal en Recién Nacidos de Clase Media». Rev.Chil.Pediatr. 64(4); 237-240, 1993. disminuir las tasas de morbi-mortalidad del RN tacneño.

2. Se deben tomar en cuenta para la clasificación del RN en GEG, AEG y PEG, los factores de corrección según paridad y talla materna y sexo fetal en todos los casos, para clasificarlos con mayor precisión.

Juez, G. y Cols.: «Crecimiento Intrauterino según Sexo Fetal y Paridad maternam. Rev.Chil.Pediatr. 60(4); 204-207, 1989.

Juez, G. y Cols.: "Influencia de la Talla Materna Sobre el Crecimiento Fetals. Rev. Chil. Obstet.Ginecol. 55(2); 104-108, 1990.

Juez, G. y Cols.: «Recién Nacidos Hospitalizados. Retardo de Crecimiento intrauterino, Proporciones Corporales». Rev. Chil. Obstet. Ginecol. 61(2); 105-107, 1996.

Juez, G. y Cols.: «Riesgo de Muerte Neonatal, Segun Curva Chilena de Crecimiento Intrauterino». Rev.Latin.Perinat. Vol.15, N11, 1995.

Juez, G.; Ventura-Junca, P.: "Crecimiento Intrauterino en un Grupo Seleccionado de Recién Nacidos Chilenos". Rev. Med. Chile. 112:759-764.1984.

Juez. G., Lucero, E.: «Recién Nacidos hospitalizados con Malformaciones Congénitas. Retardo de Crecimiento Intrauterino. Proporciones Corporales». Rev. Chil. Obstet.Ginecol. 61(2): 105-107, 1996.

Juez, G.: «Crecimiento Intrauterino en Gemelos Fisiológicos Chilenos». Rev.Méd.Chile. 124:117-118, 1995.

Juez, G.: "Características Materno-Neonatales del Retardo de Crecimiento Intrauterino". Rev. Chil.Obstet. Ginecol. 57(3):215216, 1992. .

Juez, G.; Ventura-Junca, P.; Lucero, E.; Tapia, J, Gonzales, H, Winter, A.: «Crecimiento Fetal en el Recién Nacido Chileno». Jornadas Científicas de la Escuela de Medicina». Santiago, 24 de Octubre 1988.

Lubchenco, L. y Cols.: «Intrauterine Growth as Stimated From Livebron Birth Date Data at 24 to 42 Weeks of Gestation». Pediatrics, 32: 793. 1963.

Lubchenco, L. y Cols.: "Intrauterine Growth in Length and Head Circunference as Estimated From Live Births at Gestational Ages From 26 to 42 Weeks". Pediatrics, Vol. 37, № 3 . March, 1966.

Mardones, F.; Dachs, J.; Diaz, M.: "Distribución el Peso al Nacer para cada Edad Gestacional en Chile". Rev. Chil.Pediatr. 60(3); 181-188, 1989.

Mardones-Santander, F.; Icaza, G. Diaz, M.:"Comparación de Tres Patrones de Valoración del Crecimiento Fetal". Bol. Of. Sanit.Panam. 112(4), 1992.

Martell, M.; Stol, P.; y cols.: «Atención Inmediata del Recién Nacido». Pub.Científica CLAP No 1253. Uruguay, 1992.

Muhlhausen, G.; Navarrete, C.: «Malnutrición Intrauterina: Comparación de Curvas de Crecimiento en la Detección de Alto Riesgo Neonatal». Rev. Chil.Pediatr. 64(1); 26-30, 1993. 
Neel, N.; Alvarez, J.: «Factores de Riesgo de Mainutrición Fetal en un Grupo de Madres y Neonatos Guatemaltecos». Bol.Of.Sanit.Panam. 110(2), 1991.

Pérez-Escamilla, R.; Pollitt, E.: «Causas y Consecuencias del Retraso del Crecimiento Intrauterino en América Latina». Bol.Of.Sanit.Panam. 112(6), 1992.

Rizzardini, M.; Silva, E.; Schildlow, D.: «Curva de Crecimiento Intrauterino en Recién Nacidos Chilenos». Rev. Chil.Pediatria. Vol.43, N 9-10, 1972.

Rosso, P.; Juez, G.: «Perinatal Outcome in Full-Term AdequateFor-Gestational age Infants With Normal and Low Ponderal Index». Pediatric Rev. Commum. Vol, 4; 251-256, 1991.

Sánchez, I.; Cavagnaro, F.; Tapia, L.; Juez, G.: «Relación entre Perimetro Braquial y Algunos Indicadores de Crecimiento Intrauterino». Rev.Chil.Pediatr. 59(5); 259-298, 1988.

Tapia, J. y Col.: «Manual de Neonatologia». Crecimiento Intrauterino. Editorial Mediterraneo. Santiago de Chile. 1ra.Ediciln, 1992.

Ticona, R.: «Curvas de Crecimiento Intrauterino de Tacna». El Recién Nacido. Morbi-Mortalidad. 161-183. Universidad Nacional Jorge Basadre Grohmann. 1995.

Ticona R.: "Perinatología. Prevención-Diagnóstico-Tratamiento". Universidad Jorge Basadre de Tacna. 1997.
Thomson, A.M. y Cols.: "The Assessment of Fetal Growth". J.Obstet.Gynaec. Brit. Cwith. Vol.75.pp.903-916. Sept.1968.

Usher, R. y Col.: "Intrauterine Growth of Liveborn Causasin Infants at Level: Standards Obtenied from Measurements in 7 dimensios of infants bron Between 25 and 44 Weeks os Gestation". L.Pediatrics. 74:901. 1969.

Vega, J.; Sáez, G.; Smith, M.: «Factores de Riesgo Para Bajo Peso al Nacer y Retardo de Crecimiento Intrauterino en Santiago de Chile. Rev.Méd.Chile. 121:1210-1219, 1993.

Ventura-Junca, P.; Juez, G.: "Desnutrición Intrauterina: Identificación de una nueva Población de Alto Riesgo con una Curva de Peso Chilena". Rev.Méd. Chile. 114:790-797, 1986.

Ventura-Juncá, P. Juez, G.; Lucero, E.: «Riesgo de Morbilidad y Mortalidad en Recién Nacidos de Término Según Adecuación del Peso a la Edad Gestacional». Rev.Chil.Pediatr. 66(2); 103-106, 1995.

Weldt, E.; Rosselot, S.; Tohá, D.; Andrade, C.: «Evaluación de Crecimiento Intrauterino Mediante el Peso de Nacimiento». Rev.Chil.Pediatr. 59(4); 27-269, 1988.

Williams, R.; Creasy, R.; Cunningham, G. Y Cols.: «Fetal Growth and Perinatal Viability in Californiam. Obstetrics \& Gynecology. Vol.59, №5, May 1982.

\section{GRÁFICO Nro. 1. CURVA DE CRECIMIENTO INTRAUTERINO EN RECIEN NACIDOS TACNEÑOS}

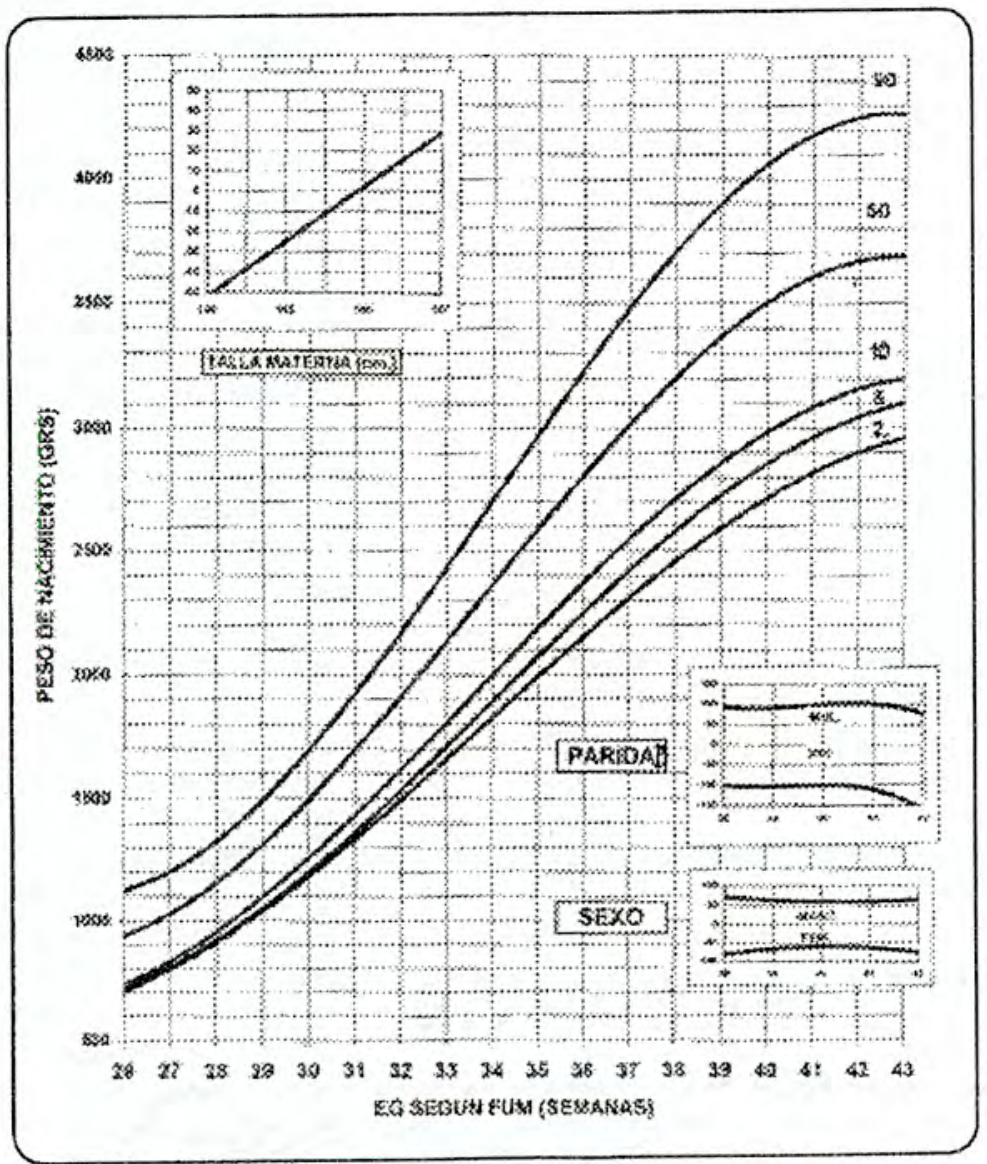


GRÁFICO Nro. 2. FACTOR DE CORRECCIÓN SEGÚN SEXO

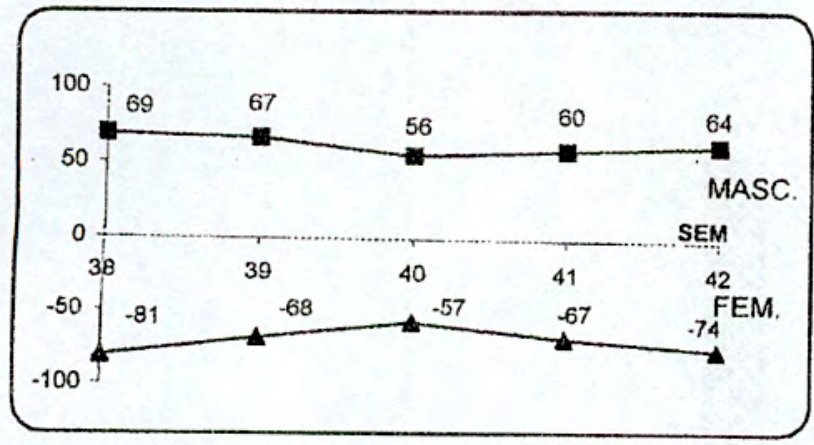

GRÁFICO Nro. 4. CURVA DE CRECIMIENTO INTRUTERINO TACNA LUBCHENCO.

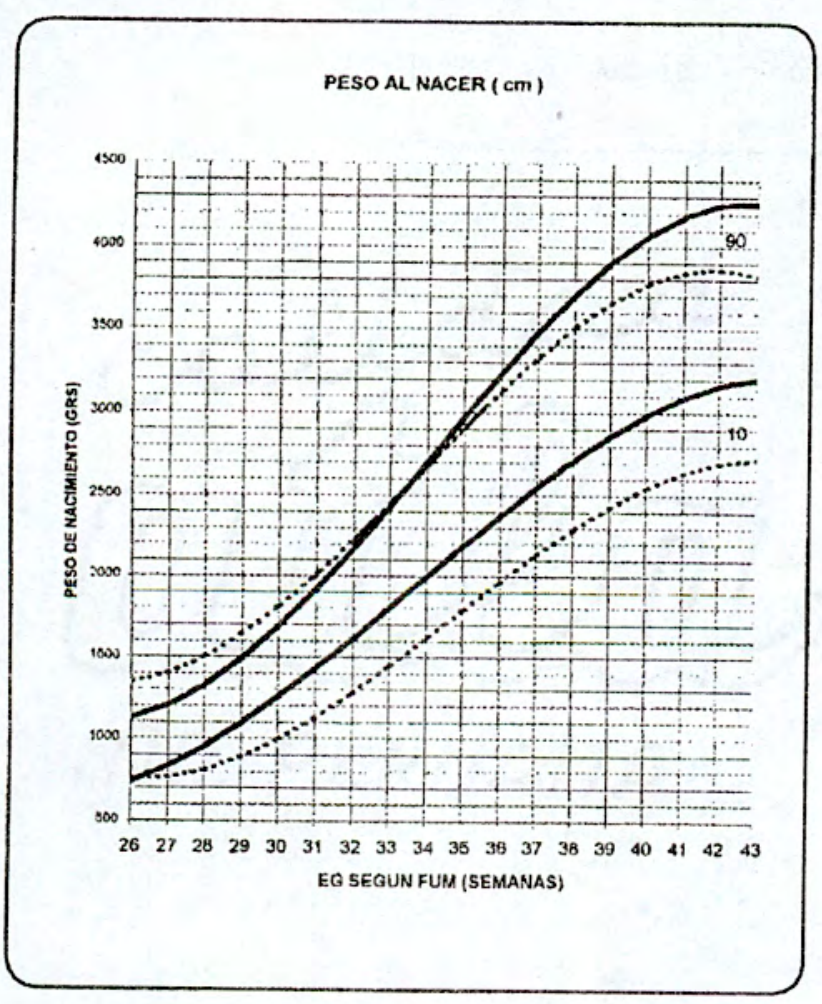

GRÁFICO NRO. 3. FACTOR DE CORRECCIÓN SEGÚN PARIDAD

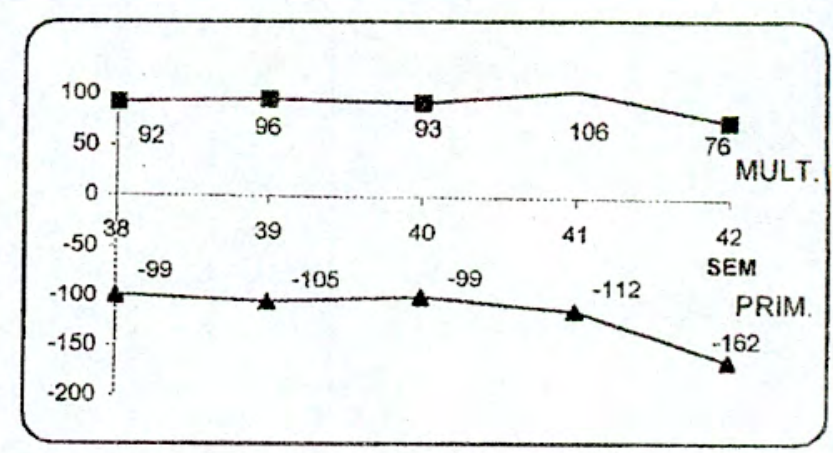

GRÁFICO Nro. 5. IDENTIFICACION DE NUEVOS PEG TACNA - LUBCHENCO.

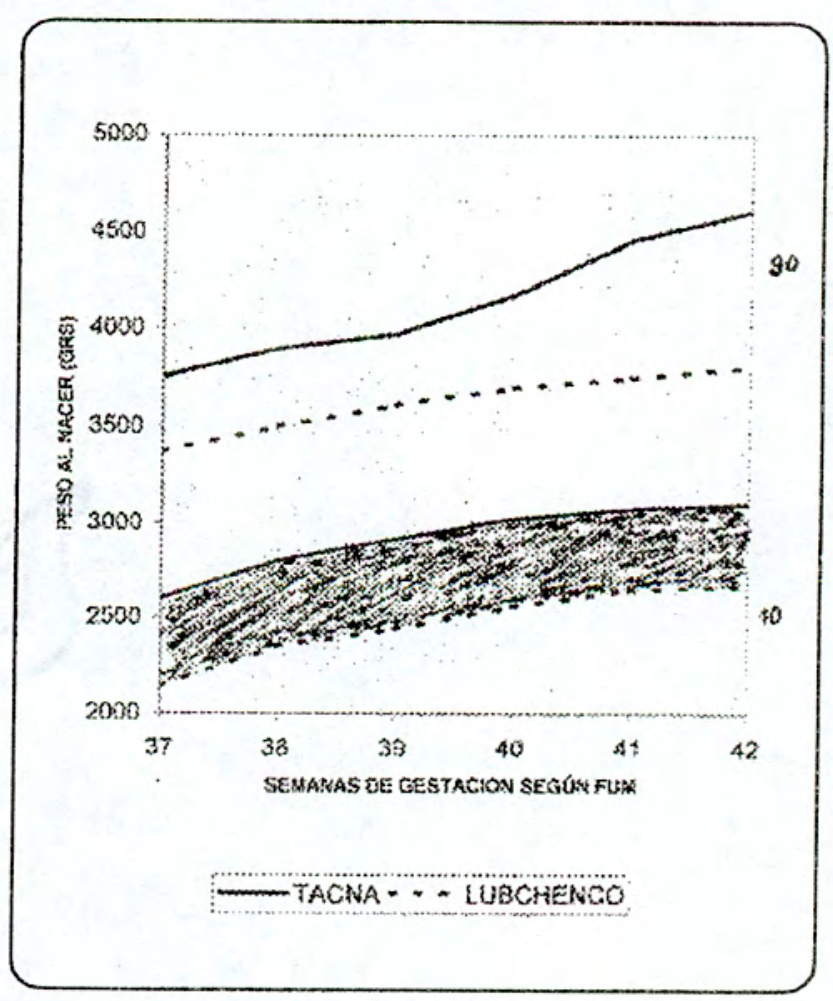


Ciencia \& Desarrollo 7

GRÁFICO Nro. 6. IDENTIFICACION DE NUEVOS PEG CON C.C.I.U. DE TACNA .

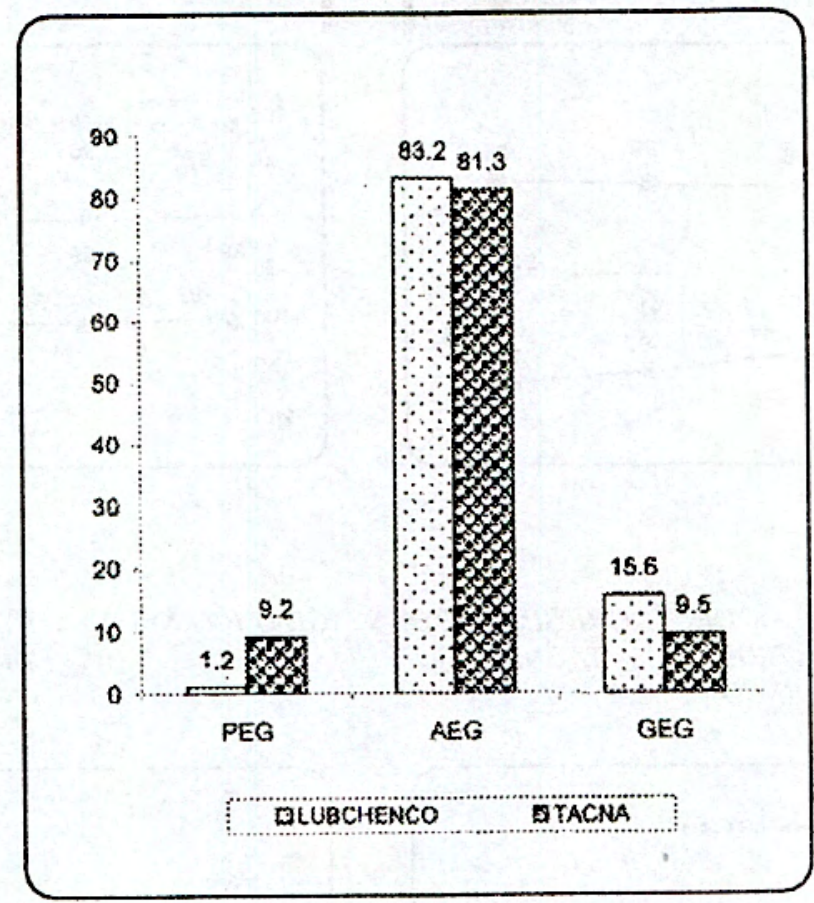

